ARTICLE

DOI: $10.1038 / s 41467-017-01934-1$

\title{
Exploration of pyrazine-embedded antiaromatic polycyclic hydrocarbons generated by solution and on-surface azomethine ylide homocoupling
}

\author{
Xiao-Ye Wang (1) 1, Marcus Richter ${ }^{2}$, Yuanqin $\mathrm{He}^{3,4}$, Jonas Björk ${ }^{5}$, Alexander Riss (10 ${ }^{4}$, Raju Rajesh ${ }^{1}$, \\ Manuela Garnica4 ${ }^{4}$ Felix Hennersdorf 6 , Jan J. Weigand ${ }^{6}$, Akimitsu Narita (1) ${ }^{1}$, Reinhard Berger (D) ${ }^{2}$, \\ Xinliang Feng ${ }^{2}$, Willi Auwärter ${ }^{4}$, Johannes V. Barth ${ }^{4}$, Carlos-Andres Palma ${ }^{4} \&$ Klaus Müllen ${ }^{1}$
}

Nanographenes, namely polycyclic aromatic hydrocarbons (PAHs) with nanoscale dimensions ( $>1 \mathrm{~nm})$, are atomically precise cutouts from graphene. They represent prime models to enhance the scope of chemical and physical properties of graphene through structural modulation and functionalization. Defined nitrogen doping in nanographenes is particularly attractive due to its potential for increasing the number of $\pi$-electrons, with the possibility of introducing localized antiaromatic ring elements. Herein we present azomethine ylide homocoupling as a strategy to afford internally nitrogen-doped, non-planar $\mathrm{PAH}$ in solution and planar nanographene on surfaces, with central pyrazine rings. Localized antiaromaticity of the central ring is indicated by optical absorption spectroscopy in conjunction with theoretical calculations. Our strategy opens up methods for chemically tailoring graphene and nanographenes, modified by antiaromatic dopants.

\footnotetext{
${ }^{1}$ Max Planck Institute for Polymer Research, Ackermannweg 10, 55128 Mainz, Germany. ${ }^{2}$ Department for Molecular Functional Materials, Center for Advancing Electronics Dresden (cfaed), Dresden University of Technology, Mommsenstr. 4, 01062 Dresden, Germany. ${ }^{3}$ Institute for Advanced Study, Technical University of Munich, Lichtenbergstr. 2a, 85748 Garching, Germany. ${ }^{4}$ Physik-Department E20, Technical University of Munich, James-Franck-Str. 1, 85748 Garching, Germany. ${ }^{5}$ Department of Physics, Chemistry and Biology, IFM, Linköping University, 58183 Linköping, Sweden. ${ }^{6}$ Chair of Inorganic Molecular Chemistry, Dresden University of Technology, Mommsenstr. 4, 01062 Dresden, Germany. Correspondence and requests for materials should be addressed to W.Aär. (email: wau@tum.de) or to J.V.B. (email: jvb@tum.de) or to C.-A.P. (email: palma@iphy.ac.cn) or to K.Mül. (email: muellen@mpip-mainz.mpg.de)
} 
T ogether with the advent of graphene science, an important chapter in organic chemistry has opened exploring the synthesis of extended polycyclic aromatic hydrocarbon (PAH) systems. Large PAHs, as represented by hexa-perihexabenzocoronene (HBC, 1) with $\pi$-conjugated structures extending over $1 \mathrm{~nm}$, can be regarded as structurally defined, nanoscale cutouts of graphene, designated nanographene molecules ${ }^{1-3}$. Such systems can serve as platforms both for investigating the physical and chemical properties of graphene upon heteroatom-doping, as well as for the development of semiconducting materials (see Fig. 1). Doped nanographenes are similarly relevant for the study of aromaticity and antiaromaticity, which are fundamental concepts of organic chemistry ${ }^{4,5}$. As proposed by Hückel ${ }^{6}$ and later by Frost and Musulin $^{7}$, as well as Breslow,$\pi$-conjugated cycles with [4n+2] $\pi$-electrons show aromatic character with strong stabilization while cycles with [4n] $\pi$-electrons are antiaromatic and destabilized. Despite a few theoretical suggestions on local antiaromatic "defects", or "dopants", in graphene $e^{9,10}$ and rapidly increasing variety of nanographene molecules, well-defined nanographene molecules with a pronounced antiaromatic ring, i.e., a local antiaromatic element, remain challenging targets to be explored.

Nitrogen $(\mathrm{N})$-doped nanographenes have attracted considerable attention due to promising electronic and magnetic properties $^{11}$ offering potential applications in metal-free catalysis ${ }^{12,13}$ and sensing ${ }^{14,15}$. However, the existing synthetic protocols do not allow the precise control of the doping level and heteroatom positioning. The chemical nature of nitrogen dopants (i.e., pyridinic, pyrrolic, and "graphitic" $\mathrm{N}$ atoms) remains largely undefined ${ }^{16}$, restricting the fine-tuning of the resulting properties as well as reliable structure-property relationship elucidation. In this regard, structurally well-defined $\mathrm{N}$-doped nanographene molecules offer an opportunity to accurately investigate $\mathrm{N}$-doping effects. Notwithstanding, N-doping of defined nanographenes has been predominantly limited to pyridinic ${ }^{17-19}$ (e.g., compound $2^{20}$ in Fig. 1) and pyrrolic (e.g., compounds $3^{21}$ and $\mathbf{4}^{22,23}$ ) $\mathrm{N}$ atoms, which only resulted in replacing benzene rings with other $\mathrm{N}$ containing $6 \pi$ aromatic rings, such as pyrimidines and pyrroles.

To develop nanographenes with strong antiaromatic ring dopants, we consider the possibility of incorporating a pyrazine structure inside the aromatic core, which employs two "graphitic" $\mathrm{N}$ atoms in the framework, thus providing additional $\pi$-electrons when compared to a benzene ring. 1,4-Disubstituted pyrazine entails intriguing chemistry, expressing $8 \pi$ antiaromatic (neutral), $7 \pi$ non-aromatic (cationic), or $6 \pi$ aromatic (dicationic) properties, depending on its oxidation state. Thus, a pyrazine ring embedded in a carbonaceous hexagonal structure serves as a localized antiaromatic ring dopant, influencing ring and perimeter currents ${ }^{24,25}$ with the potential to give rise to new types of 2D materials with unconventional semiconducting properties.

Here, we report in situ solution synthesis and spectroscopy of the partially fused, pyrazine-incorporated hexabenzoperylene (HBP) derivative 6a as well as on-surface synthesis of the diazaHBC $7 c$ with a completely embedded pyrazine core. N-doped HBP 6a features an $8 \pi$ antiaromatic core structure, as documented by ${ }^{1} \mathrm{H}$ NMR and UV-vis absorption spectroscopy together with density functional theory (DFT) calculations. Notably, the solution chemistry of polycyclic aromatic azomethine ylides (PAMYs) ${ }^{26}$ can be successfully adapted on the $\operatorname{Ag}(111)$ surface, achieving the synthesis of $\mathbf{7 c}$ through surface-assisted homocoupling of the dibenzo-9a-azaphenalene (DBAP) salt $\mathbf{5 c}$ and concomitant cyclodehydrogenation. High-resolution scanning

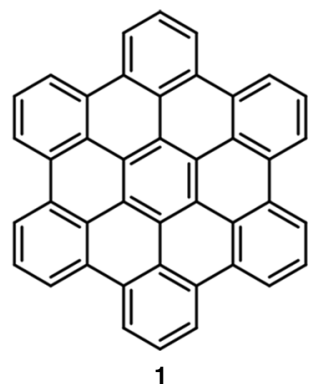

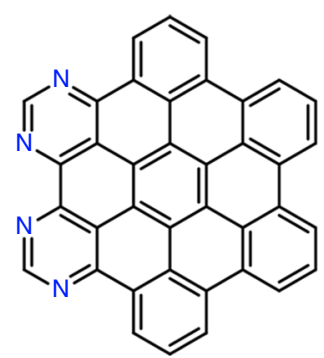

2

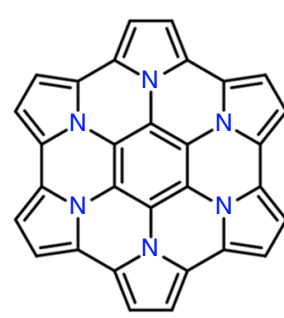

3

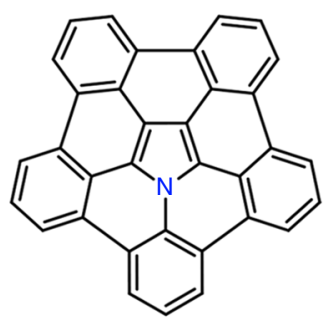

4

Fig. 1 Chemical structures of pristine and N-doped nanographenes. Hexa-peri-hexabenzocoronene (HBC) 1, periphery-doped tetraaza-HBC $\mathbf{2}$, azacoronene derivatives $\mathbf{3}$, and azacorannulene $\mathbf{4}$

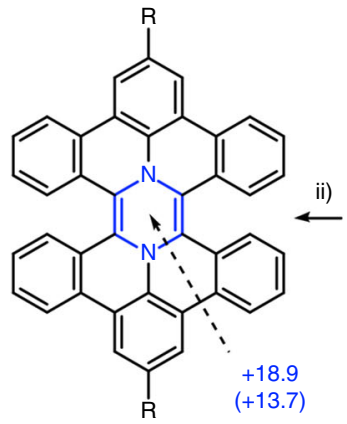

6a: $\mathrm{R}=\mathrm{OMe}$

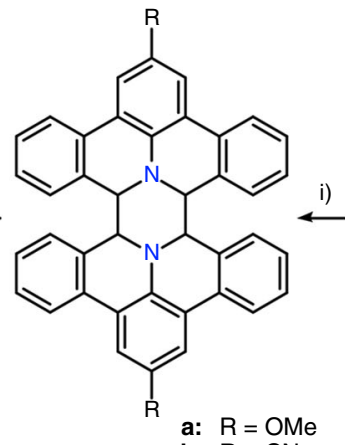

8 b: $R=C N$

c: $\mathrm{R}=\mathrm{H}$

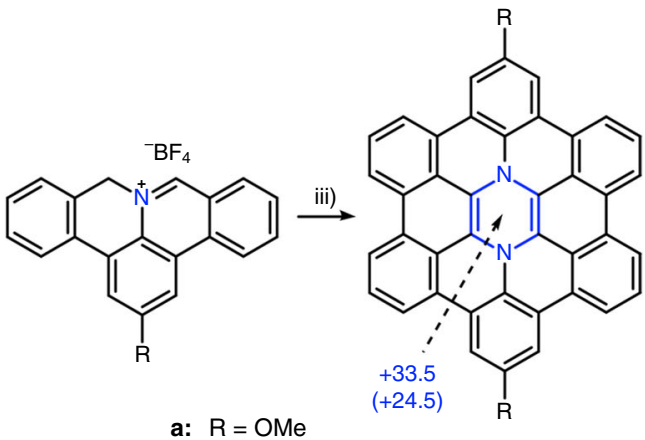

7c: $\mathrm{R}=\mathrm{H}$

Fig. 2 Synthesis of diaza-HBP $\mathbf{6 a}$ in solution and internally N-doped diaza-HBC 7c upon interfacial confinement. Reagents and conditions: (i) tributylamine, DMSO, $190{ }^{\circ} \mathrm{C}$; (ii) DDQ, $\mathrm{C}_{2} \mathrm{D}_{2} \mathrm{Cl}_{4}, 100^{\circ} \mathrm{C}$; (iii) in vacuo on the $\mathrm{Ag}(111)$ surface, $270{ }^{\circ} \mathrm{C}$. The calculated nucleus-independent chemical shifts (NICS) values are depicted in blue (unit: ppm), indicating the antiaromatic nature of the pyrazine ring (in parenthesis the NICS(1) value, see text and Supplementary Fig. 1) 
tunneling microscopy (STM) and frequency-modulated atomic force microscopy (FM-AFM) ${ }^{27}$ unambiguously elucidate the chemical structure of diaza-HBC $7 \mathrm{c}$. Moreover, computational modeling using DFT indicates that $7 \mathrm{c}$ conserves its $8 \pi$ character on the $\operatorname{Ag}(111)$ surface. Nucleus-independent chemical shift $(\text { NICS })^{28}$ calculations on $6 \mathbf{a}$ and $7 \mathrm{c}$ reveal high positive values at the center and $1 \AA$ above the center of the central pyrazine rings (NICS(0) and NICS(1) values, respectively), indicative of anti-

a

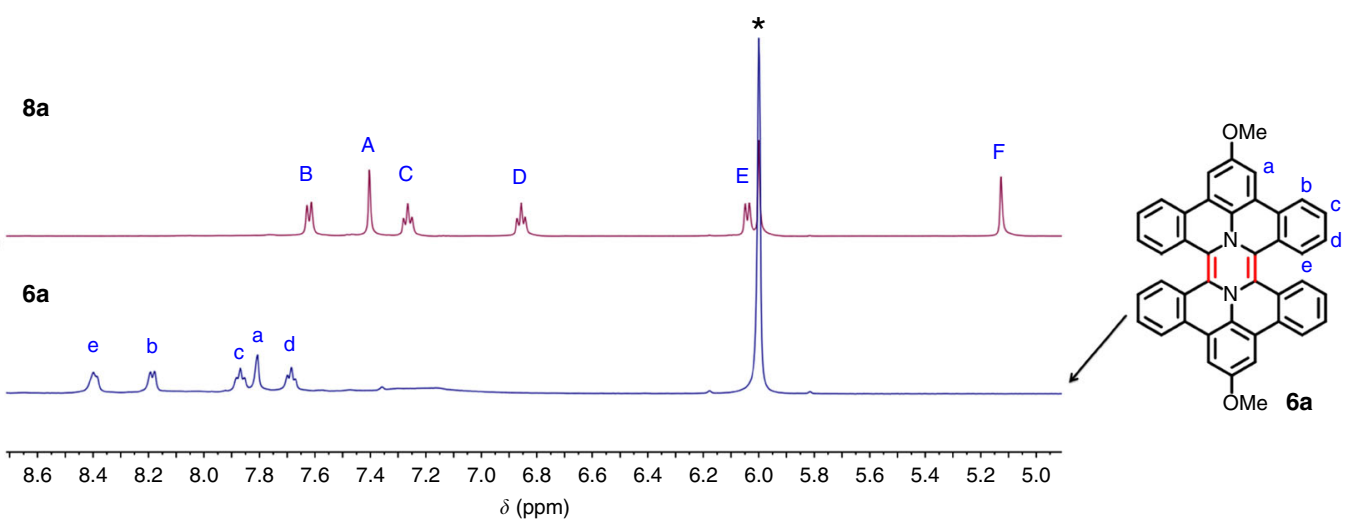

b

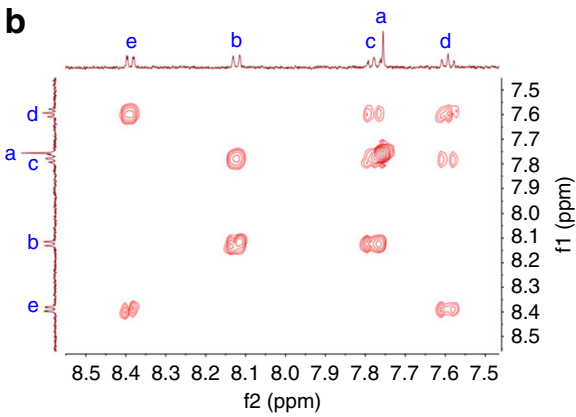

c

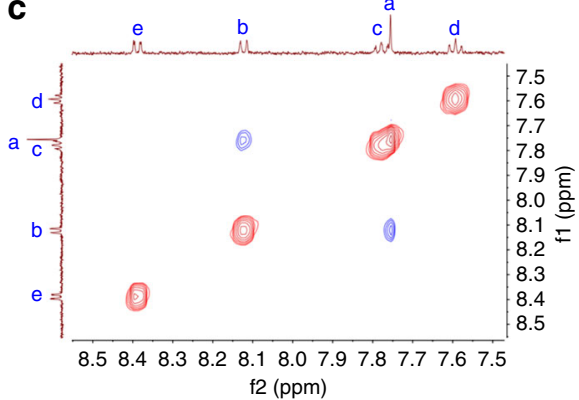

d

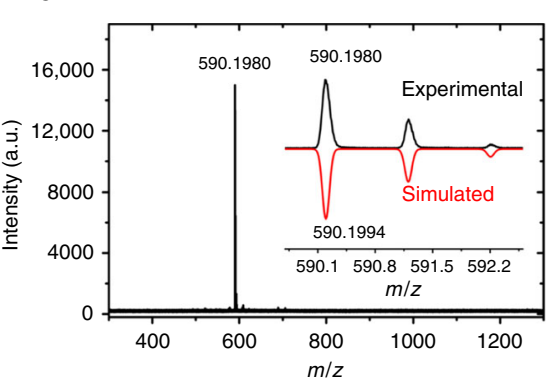

Fig. 3 Structural characterizations of the in situ generated diaza-HBP $\mathbf{6 a} \cdot \mathbf{a}{ }^{1} \mathrm{H}$ NMR spectra of $\mathbf{8} \mathbf{a}$ and $\mathbf{6 a}$ together with their structural assignments $\left({ }^{\star} \mathrm{C}_{2} \mathrm{HDCl}_{4}\right)$. Compared to precursor $\mathbf{8} \mathbf{a}$, the signals of $\mathbf{6} \mathbf{a}$ were down-field shifted, corroborating the extension of the $\pi$-conjugation. $\mathbf{b}$ Aromatic region of the ${ }^{1} \mathrm{H}-{ }^{1} \mathrm{H}$ COSY spectrum of $\mathbf{6} \mathbf{a}$ in $\mathrm{C}_{2} \mathrm{D}_{2} \mathrm{Cl}_{4}$. c Aromatic region of the ${ }^{1} \mathrm{H}-{ }^{1} \mathrm{H}$ NOESY spectrum of $\mathbf{6} \mathbf{a}$ in $\mathrm{C}_{2} \mathrm{D}_{2} \mathrm{Cl}_{4}$. $\mathbf{d}$ High-resolution MALDI-TOF MS spectrum of $\mathbf{6 a}$, with an inset showing its experimental (black line) and simulated isotopic distributions (red line)

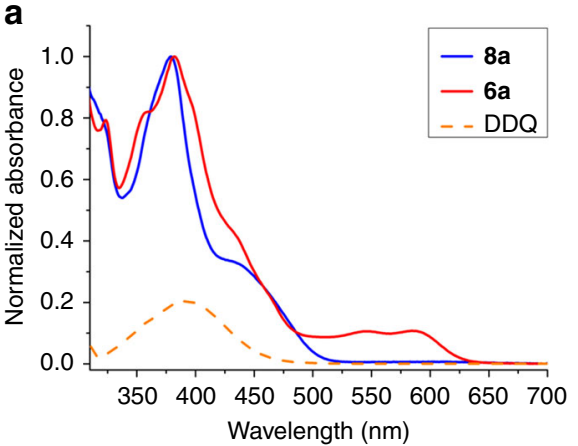

C

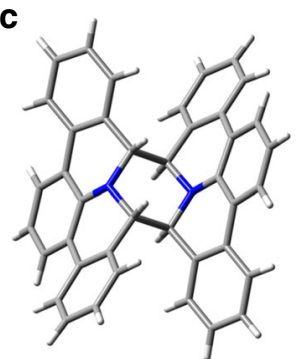

$8 \mathrm{c}$ d

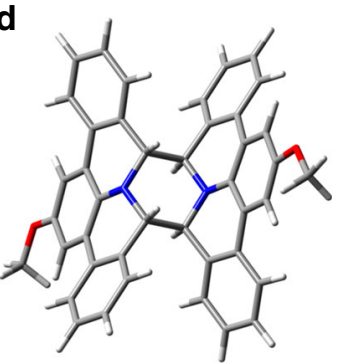

$8 \mathbf{a}$

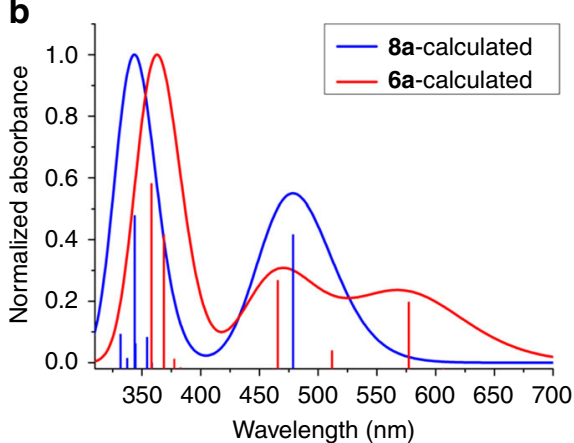

$\mathbf{e}$

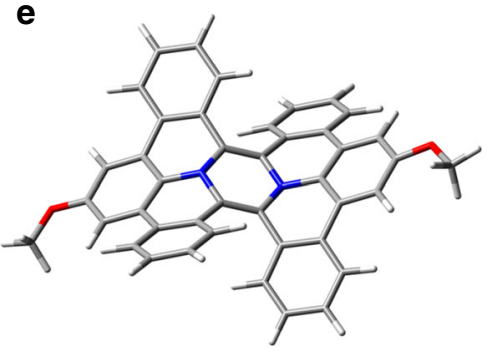

$6 \mathbf{a}$

Fig. 4 Photophysical investigations. a Experimental and $\mathbf{b}$ TDDFT-simulated absorption spectra of $\mathbf{6} \mathbf{a}$ and its precursor $\mathbf{8 a}$. The simulated spectra of $\mathbf{6 a}$ showed the appearance of a new band at $577 \mathrm{~nm}$, in accordance with the experimental observation. $\mathbf{c}$ X-ray single-crystal structure of $\mathbf{8 c}$. $\mathbf{d}$ DFT-optimized structure of $\mathbf{8 a}$. e DFT-optimized structure of $\mathbf{6 a}$ 

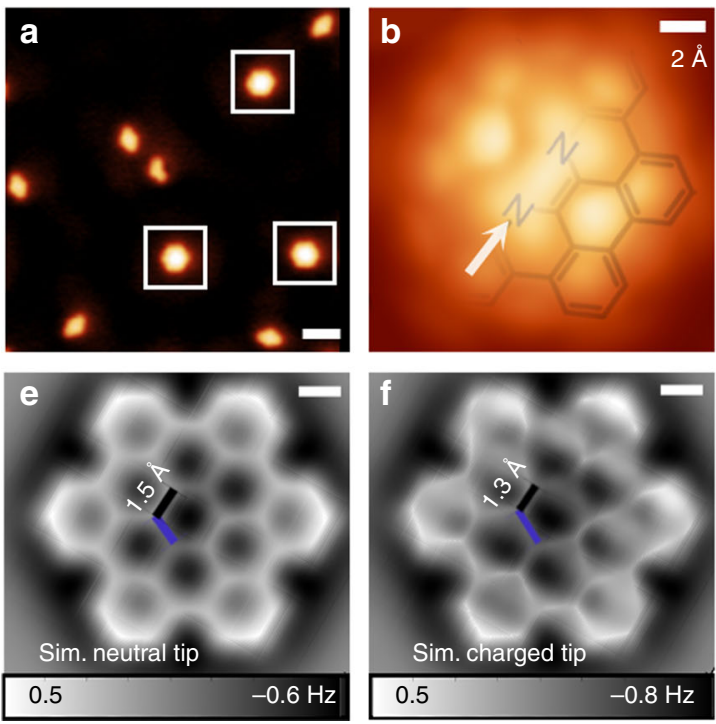
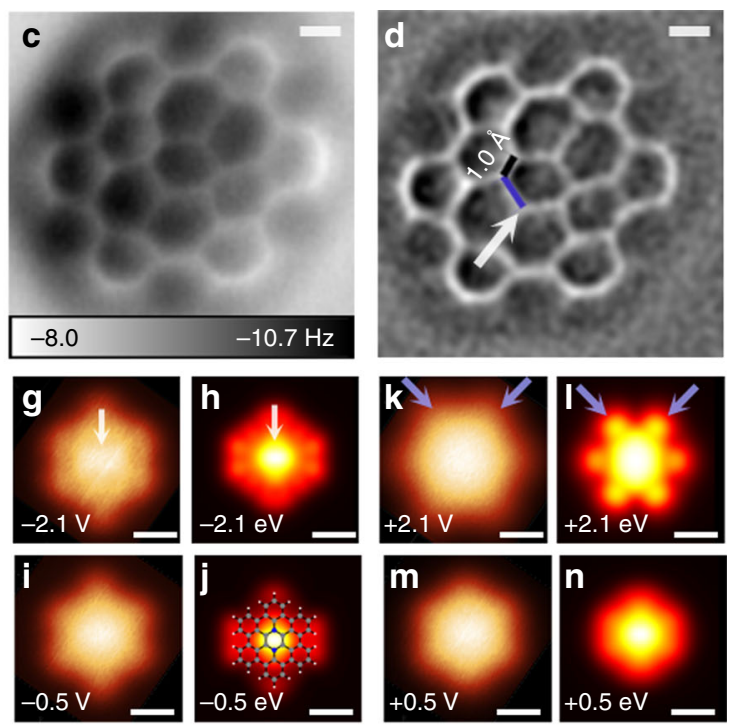

Fig. 5 On-surface homocoupling of precursor 5c yields fully planar and neutral diaza-HBC 7c on Ag(111). a Scanning tunneling microscopy (STM) overview of reaction product and unreacted species on $\mathrm{Ag}(111)$. $V_{\mathrm{s}}=30 \mathrm{mV}, \mathrm{I}_{\mathrm{t}}=10 \mathrm{pA}$. b Constant-height STM data of diaza-HBC with partially superposed molecular model. $V_{s}=5 \mathrm{mV}$. c Frequency-modulated atomic force microscopy (FM-AFM) and d Laplace-filtered FM-AFM data reveal details in the

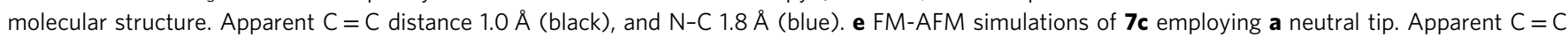
distance $1.5 \AA$ (black), N-C $1.5 \AA$ (blue). f FM-AFM simulations employing a negatively charged probe tip. Apparent C= C distance $1.3 \AA$ (black), N-C $1.9 \AA$ (blue). For simulation parameters see Methods. $\mathbf{g}-\mathbf{n}$ Constant-height STM data (g, i, $\mathbf{k}, \mathbf{m}$ ) at different biases and corresponding (h, j, I, $\mathbf{n}$ ) DFT simulations. White arrows point to $\mathrm{N}$ atoms oriented along the diaza-HBC symmetry axis. Scale bars (a) $20 \AA$, (b-f) $2 \AA$, (g-n) $5 \AA$

aromaticity. These results demonstrate the antiaromatic nature of pyrazine-doped nanographene molecules and provide an onsurface synthesis route to $\mathrm{N}$-doping nanographene materials.

\section{Results}

Solution synthesis and characterizations. The synthesis of pyrazine-embedded HBP 6a (Fig. 2) was carried out based on the chemistry of DBAP, which has been employed to construct $\mathrm{N}$ doped PAHs such as dibenzo[ $[d, k]$ ullazines and $6 b^{2}$-azapentabenzo $[b c, e f, h i, k l, n o]$ corannulenes ${ }^{22,26,29,30}$. DBAP salts 5a-c with different substituents were first synthesized according to our previous $\operatorname{method}^{26}$. They were then dimerized to form precursors 8a-c (Fig. 2) by treatment with a large excess of tributylamine in dry dimethyl sulfoxide (DMSO) under argon at $190^{\circ} \mathrm{C}$. Oxidation of 8a with excess 2,3-dichloro-5,6-dicyano-1,4-benzoquinone (DDQ) in dry $\mathrm{C}_{2} \mathrm{D}_{2} \mathrm{Cl}_{4}$ afforded diaza-HBP $\mathbf{6 a}$, as characterized by ${ }^{1} \mathrm{H}$ NMR and mass spectroscopy (MS) analyses (Fig. 3). Diaza-HBP 6a turned out to be extremely unstable probably because of the antiaromatic pyrazine core, and thus the oxidation step was performed in situ in a sealed NMR tube under exclusion of air. Oxidation of other precursors $\mathbf{8 b}$ and $\mathbf{8 c}$ were also attempted with the same method, but failed to provide corresponding diaza-HBP derivatives according to ${ }^{1} \mathrm{H}$ NMR analysis, suggesting that the electrondonating methoxy groups of $\mathbf{8 a}$ played an important role in the reaction $^{31}$. As shown in Fig. 3a, the disappearance of the characteristic signal from $\mathrm{H}_{\mathrm{F}}(5.13 \mathrm{ppm})$ of $\mathbf{8 a}$ indicated complete conversion of the starting material. All the signals in the aromatic region of $\mathbf{6 a}$ were down-field shifted compared to those of $\mathbf{8 a}$, in agreement with the extension of the $\pi$-conjugation upon dehydrogenation. Notably, proton $\mathrm{H}_{\mathrm{E}}$ of $\mathbf{8 a}$ is above the neighboring benzene plane according to the optimized geometry by DFT (Fig. 4) and thus is strongly shielded. In contrast, after dehydrogenation of 8a via $D D Q$, the proton $\mathrm{H}_{e}$ of $\mathbf{6 a}$ is shifted away from the neighboring benzene into its deshielding region, in accordance with a down-field shift of $\mathrm{H}_{\mathrm{e}}$ in the ${ }^{1} \mathrm{H}$ NMR spectrum. Moreover, ${ }^{1} \mathrm{H}-{ }^{1} \mathrm{H}$ correlation spectroscopy (COSY) and ${ }^{1} \mathrm{H}_{-}{ }^{1} \mathrm{H}$ nuclear Overhauser effect spectroscopy (NOESY) analyses (Fig. 3b, c) allowed unambiguous assignments of the proton signals of $\mathbf{6 a}$. The structure was further characterized by ${ }^{1} \mathrm{H}_{-}{ }^{13} \mathrm{C}$ heteronuclear single-quantum correlation (HSQC) and ${ }^{1} \mathrm{H}-{ }^{13} \mathrm{C}$ heteronuclear multiple-bond correlation (HMBC) spectroscopies. The chemical identity of $\mathbf{6 a}$ was confirmed by high-resolution matrix-assisted laser desorption/ ionization time-of-flight (MALDI-TOF) MS, which showed a matching isotopic pattern starting at $\mathrm{m} / z=590.1980$, in agreement with the theoretical value of 590.1994 (Fig. 3d).

To investigate the photophysical properties of $\mathbf{6 a}$, we prepared diaza-HBP $\mathbf{6 a}$ in situ by carrying out the oxidation reaction in a sealed quartz cell and recorded the absorption and photoluminescence spectra. As shown in Fig. 4a, dimer precursor 8a exhibited an absorption maximum at $\lambda_{\max }=390 \mathrm{~nm}$, and an absorption onset at $\approx 500 \mathrm{~nm}$. After dehydrogenation, the absorption maximum barely changed, but a new band emerged in the range of 500 to $630 \mathrm{~nm}$, suggesting an extended $\pi$-conjugation. To further understand the photophysical properties, DFT calculations were performed on both $\mathbf{8 a}$ and $\mathbf{6 a}$. In order to determine the exact molecular geometry of $\mathbf{8 a}$, growth of single crystals of $\mathbf{8 a - c}$ was pursued and a single crystal of $\mathbf{8 c}$ suitable for X-ray analysis was successfully obtained, revealing an anti-folded structure (Fig. 4c). Thus, the anti-folded geometry was employed as input geometry for the DFT optimization of similar precursors (Fig. 4d). DFT studies on dehydrogenated $\mathbf{6 a}$ indicate that the anti-folded conformation (Fig. 4e) is energetically preferred compared to the twisted one. Time-dependent DFT (TDDFT) calculations of $\mathbf{6 a}$ and $8 \mathbf{a}$ indicated that the emerging absorption band of $6 \mathbf{a}$ can be attributed to the $\mathrm{HOMO} \rightarrow \mathrm{LUMO}+2$ transition (Fig. $4 \mathrm{~b}$ and Supplementary Tables 1-3). The HOMO $\rightarrow$ LUMO transition of $\mathbf{6 a}$ is symmetry forbidden, and $\mathbf{6 a}$ is non-emissive, in agreement with previous reports on antiaromatic systems ${ }^{32,33}$.

On-surface synthesis of 7c. Despite generation of diaza-HBP 6 a through solution synthesis, further characterization of $\mathbf{6 a}$ was restricted by its low stability. In addition, attempts to oxidize intermediate product $\mathbf{8 a - c}$ directly to diaza-HBC derivatives resulted in insoluble solids, which could not be characterized. 

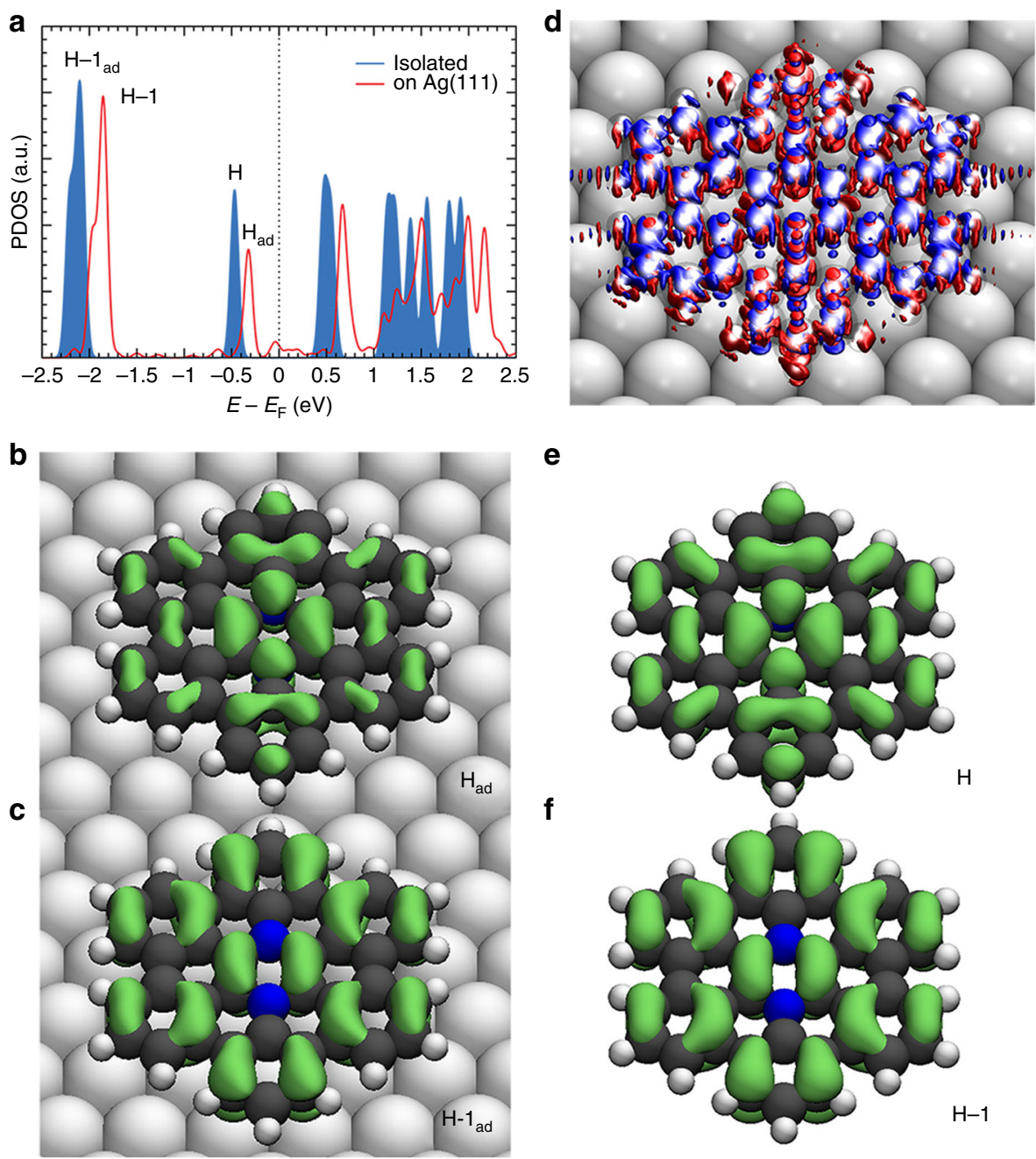

e

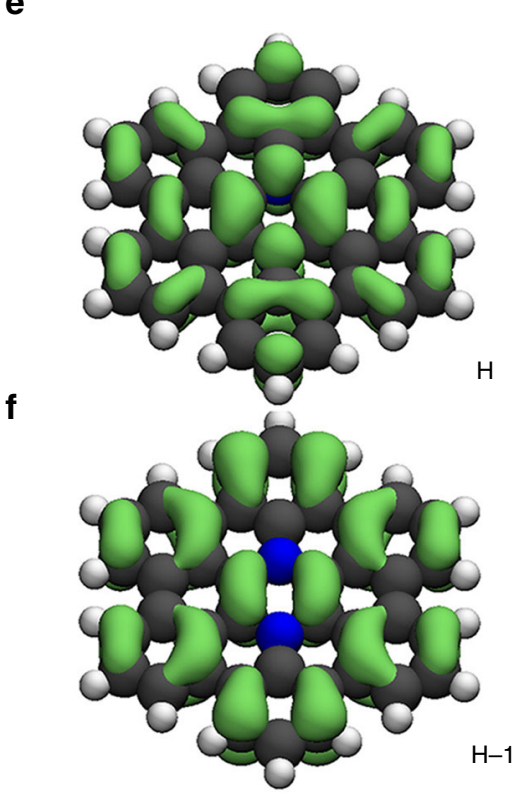

Fig. 6 DFT electronic structure comparison of the isolated and $\mathrm{Ag}(111)$-supported diaza-HBC 7c. a Projected $p_{\mathrm{z}}$ contributions to the density of states of isolated 7c (blue) and on $\mathrm{Ag}(111)$. The Fermi energy $\left(E_{\mathrm{F}}\right)$ of the isolated molecule has been arbitrarily defined for comparison. $\mathbf{b}$, c Contribution of the highest occupied molecular orbital $\mathbf{H}_{\mathbf{a d}}$ (at $-0.32 \mathrm{eV}$ in $\mathbf{a}$ ) and (H-1) ad to $\mathbf{7 c}$ on $\mathrm{Ag}(111)$. $\mathbf{d}$ Electron density difference between $\mathbf{7 c}$ on $\mathrm{Ag}(111)$ and the isolated molecule plus isolated substrate. Red regions represent electron density depletion, whilst blue electron density gain. Atoms are rendered translucent. e, $\mathbf{f}$ Highest occupied molecular orbital $\mathbf{H}$ and $\mathbf{H - 1}$ of isolated $\mathbf{7 c}$. These results suggest that antiaromatic character is preserved on $\mathrm{Ag}(111)$

This led us to adapt the solution chemistry of PAMYs to onsurface synthesis ${ }^{34-37}$, which offers today a new approach to organic chemistry. We thus deposited $\mathbf{5 c}$ on $\mathrm{Ag}(111)$ by molecular beam evaporation (Methods section and Supplementary Fig. 2). Upon annealing to $270{ }^{\circ} \mathrm{C}$, small quantities of diaza-HBC $7 \mathbf{c}$ were detected as displayed in Fig. 5a. Unlike solution synthesis (Fig. 2), no intermediate product $\mathbf{8 c}$ was identified after annealing the deposited $\mathbf{5 c}$ in several ultra-high vacuum (UHV) preparations on $\mathrm{Ag}(111), \mathrm{Ag}(100), \mathrm{Cu}(111)$, and boron nitride (BN) on $\mathrm{Cu}(111)$, suggesting that homocoupling and dehydrogenation occur synergistically at the employed reaction temperatures. Notably, $7 \mathbf{c}$ was detected on $\mathrm{BN} / \mathrm{Cu}(111)$ templates ${ }^{38}$ at similar annealing temperatures in trace-quantities (Supplementary Fig. 3), indicating that the on-surface coupling strategy proceeds in a metal-free fashion, further motivating studies on reaction mechanisms and on-surface synthesis on insulators.

High-resolution, constant height STM (Fig. 5b) and FM-AFM (Fig. $5 \mathrm{c}, \mathrm{d}$ ), using $\mathrm{CO}$-functionalized tips, confirmed $7 \mathrm{c}$ on $\mathrm{Ag}$ (111). In FM-AFM data on $\mathrm{Ag}(111)$, the central pyrazine ring of 7c (Fig. 5d) appears distorted. Observation of a $D_{2 h}$ symmetry points toward the nitrogen atoms sitting at the positions indicated by white arrows. Note that the predicted DFT $\mathrm{C}=\mathrm{C}$ bond length of the central pyrazine ring is $1.40 \AA$ vs. $1.42 \AA$ for the $\mathrm{N}-\mathrm{C}$ bond, and the molecule remains planar $(z \pm 0.05 \AA)$ for calculated adsorption sites on $\mathrm{Ag}(111)$ (Supplementary Fig. 4). Bond lengths in AFM data typically undergo distorsions ${ }^{27,39-41}$ especially under the influence of electrostatic interactions with the $\mathrm{CO}$ tip $^{42-46}$. This causes a distorted appearance of the central pyrazine ring. Quantitative evidence of these combined effects is summarized in the AFM frequency shift simulation including a DFT-derived electrostatic potential (Fig. 5e, f, see Methods section). Note how the apparent $\mathrm{C}=\mathrm{C}$ bond length in the simulated image $(1.3 \AA)$ appears shorter than the apparent $\mathrm{N}-\mathrm{C}$ bond length $(1.9 \AA$, Fig. $5 f)$, in good agreement with the experimental data (1.0 and $1.8 \AA$, respectively). When a neutral tip is employed for the AFM simulation the central pyrazine ring is fully symmetric, with apparent $\mathrm{N}-\mathrm{C}$ and $\mathrm{C}=\mathrm{C}$ lengths of $1.5 \AA$ (Fig. 5e), confirming that the distortions in the FM-AFM data originate mostly from electrostatic effects.

Combined scanning probe data and theoretical investigations indicate that $7 \mathrm{c}$ remains neutral (conserving its pyrazine $8 \pi$ state) on the surface, which is a requirement for antiaromaticity. Bader charge analysis was employed to assess molecule-substrate charge transfer. As shown by NICS calculations on isolated molecules, a neutral species of $7 \mathrm{c}$ implies an $8 \pi$ pyrazine unit, which is antiaromatic in the isolated state. Upon oxidation, a positively 
charged " $7 \pi$ " radical cation state of the diaza-HBC $7 \mathrm{c}$ displays significantly reduced antiaromaticity and a " $6 \pi$ " dication species exhibits an aromatic character (Supplementary Fig. 1). On Ag (111), theoretical charge analysis finds only marginal electron transfer to the substrate (the molecule is charged positively by less than $+0.14 e$ for all calculated molecular configurations on $\mathrm{Ag}$ (111), Supplementary Fig. 4). Moreover, when trying to enforce molecular charging by removing electrons from the calculated system, the charge at the molecule was only increased to about + $0.8 e$, when the whole $29 \AA \times 25 \AA \times 22 \AA$ unit cell was charged by $+10 e$. These theoretical analyses indicate that $7 \mathrm{c}$ remains in the neutral " $8 \pi$ " state on $\mathrm{Ag}(111)$. The good correlation of the STM experimental data with Tersoff-Hamann simulations supports the validity of the theoretical conclusions (Fig. $5 \mathrm{~g}, \mathrm{n}$ and see Supplementary Fig. 5 for extended comparisons). It should be noted how at negative energies (voltages), the symmetry of the tunneling image follows the diaza-HBC $7 \mathrm{c}$ hexagonal frame (marked by a white arrow, Fig. 5g, h). At high positive biases (between 0.9 and $2.1 \mathrm{eV}$, see Supplementary Fig. 5) the symmetry of the tunneling data is rotated (blue arrows, Fig. 5k, l) with respect to the diaza-HBC hexagonal frame, that is, tunneling is enhanced between the bay regions in the periphery of diaza-HBC. Notably, between -0.5 and $0.5 \mathrm{eV}$ (Fig. 5i, j, m, n), both experiment and simulation follow the hexagonal symmetry of $7 \mathrm{c}$ on $\operatorname{Ag}(111)$.

Having identified a neutral state of $7 \mathbf{c}$ on $\operatorname{Ag}(111)$, comparison between $7 \mathrm{c}$ on $\operatorname{Ag}(111)$ and the isolated antiaromatic species provides evidence on the preserved antiaromatic character of $7 \mathbf{c}$ on $\operatorname{Ag}(111)$. Figure 6 depicts the site-projected density of states (PDOS) as well as plots for the individual Kohn-Sham orbitals. Only marginal hybridization resulting in mid-gap states is observed when the isolated molecule interacts with $\operatorname{Ag}(111)$ (Fig. 6a, red line). The mid-gap states do not appear to significantly influence the antiaromatic character of the adsorbed molecule: the HOMO $(\mathrm{H})$ and HOMO-1 (H-1) signatures of isolated $7 \mathbf{c}$ are reflected undisturbed at the surface (compare Fig. $4 b, c, e, f)$. Instead, the electronic density difference plot (Fig. 6d) shows that the mid-gap states originate from homogeneous orbital contributions, including the role of rings which are considered to be aromatic (Supplementary Fig. 1). Note that scanning tunneling spectroscopy follows the major theoretical LDOS features including a pronounced broadening (Supplementary Fig. 6).

\section{Discussion}

In summary, we have demonstrated the chemical synthesis of a partially fused PAH in solution and the on-surface synthesis of a fully planarized nanographene molecule with antiaromatic pyrazine dopants. Diaza-HBP 6a has been achieved in solution and investigated by NMR, high-resolution MALDI-TOF MS and UVvis absorption spectroscopy, while the fully cyclized diaza-HBC $7 \mathbf{c}$ has been successfully fabricated on $\operatorname{Ag}(111)$ and characterized by STM and FM-AFM. We suggest that pyrazine rings are potential candidates to be included in $2 \mathrm{D}$ materials to spawn novel functional properties induced by breaking the conjugated lattice via antiaromatic ring dopants. Importantly, the chemistry of on-surface azomethine ylide homocoupling reported here opens up a new route to novel nanographene materials, especially graphene nanoribbons, on a large variety of substrates.

\section{Methods}

Procedure for the in situ preparation of 6a. Inside an argon-filled glovebox, precursor $8 \mathbf{a}\left(2.0 \mathrm{mg}, 1.0\right.$ eq.), DDQ (15 mg, 20 eq.) and $\mathrm{C}_{2} \mathrm{D}_{2} \mathrm{Cl}_{4}(0.6 \mathrm{~mL})$ were added sequentially into a sealable NMR tube. The mixture was heated at $100^{\circ} \mathrm{C}$ for $1 \mathrm{~min}$ and cooled down to room temperature, and then analyzed by NMR spectroscopy. Synthetic procedures and characterization data of $\mathbf{8 a - c}$ and $\mathbf{6 a}$ are provided in the Supplementary Methods and Supplementary Figs. 7-45.

STM and FM-AFM measurements. Precursor $5 \mathbf{c}$ was sublimated between $200^{\circ} \mathrm{C}$ and $220^{\circ} \mathrm{C}$ at $10^{-9}$ mbar. Data was acquired in situ using a CreaTec STM/AFM $(5.5 \mathrm{~K})$ under ultra-high vacuum $\left(10^{-10} \mathrm{mbar}\right)$. AFM data were taken in constant height mode at $0.0 \mathrm{~V}$ bias, using a qPlus sensor (resonance frequency $\approx 22 \mathrm{kHz}$, oscillation amplitude: $70 \mathrm{pm}$ to $100 \mathrm{pm}$ ). The tungsten tip was prepared by focused-ion beam processing and in situ tip forming. For subsequent tip functionalization, carbon monoxide (CO) was dosed on the cold sample and transferred to the tip. The images were processed using the Gwyddion software package ${ }^{47}$.

Computational details. DFT Calculations in Fig. 4 were performed using the Gaussian 09 software package. The geometries were optimized at the B3LYP/6-311 $\mathrm{G}(\mathrm{d}, \mathrm{p})$ level. The excitation energies were calculated by the TDDFT method at the B3LYP/6-311 G(d,p) level. NICS were calculated using the gauge invariant atomic orbital (GIAO) approach at the GIAO-B3LYP/6-311 + G(2d,p) level. NICS(1) values were averaged by two positions (above and below the plane). For Figs. 5 and 6 , periodic DFT calculations were performed with the VASP $\operatorname{code}^{48}$, projector augmented wave potentials ${ }^{49}$ and plane waves expanded to a kinetic energy cutoff of $400 \mathrm{eV}$. Exchange-correlation effects were treated by the van der Waals density functional ${ }^{50}$ in the version denoted as rev-vdWDF $2^{51}$. The $\mathrm{Ag}(111)$ surface was represented by four layered slab separated by approx. $15 \AA$ of vacuum region. Furthermore, a $p(10 \times 10)$ super cell was used together with a $2 \times 2 k$-point sampling All structures were geometrically optimized until the residual forces on all atoms were smaller than $0.01 \mathrm{eV} / \AA$, except for the bottom two layers of the slab, which were frozen in the bulk distance. An exhaustive conformational search identified the preferred adsorption site (Site b, Supplementary Fig. 4). The site's adsorption height was further optimized for a gas-phase molecule and found to be $3.22 \AA$ between the molecular and metallic planes (Site c, Supplementary Fig. 4). This site was employed to simulate the STM and AFM images. STM images were simulated with the Tersoff-Hamann approximation ${ }^{52}$ using the implementation by Lorente and Persson ${ }^{53}$. AFM simulations were performed with a modification of the particle probe model code ${ }^{42}$ by Hapala et al. The DFT-derived Hartree potential was employed to prepare an electrostatic grid which together with the molecular Lennard-Jones potential acted as the force-field for simulating the frequency shift of a probe $\mathrm{C}=\mathrm{O}$ particle as detailed in ref. ${ }^{46}$. The simulations involved oscillating a probe particle with an amplitude of $5 \AA$ at a height of $7.7 \AA$ above the substrate. The terminal oxygen was given a negative charge of $-0.005 e$. The probe's stiffness $x, y, z$ components were set to $3 \mathrm{~N} \mathrm{~m}^{-1}, 3 \mathrm{~N} \mathrm{~m}^{-1}$ and $50 \mathrm{~N} \mathrm{~m}^{-1}$, respectively.

Data availability. X-Ray crystallographic data can be obtained free of charge from the Cambridge Crystallographic Data Centre (CCDC) with the CCDC number 1529624 via its website (https://www.ccdc.cam.ac.uk/structures/). All other data are available within the article and its Supplementary Information or from the corresponding authors upon reasonable request.

Received: 14 February 2017 Accepted: 26 October 2017

Published online: 05 December 2017

\section{References}

1. Narita, A., Wang, X. Y., Feng, X. L. \& Müllen, K. New advances in nanographene chemistry. Chem. Soc. Rev. 44, 6616-6643 (2015).

2. Cai, J. M. et al. Atomically precise bottom-up fabrication of graphene nanoribbons. Nature 466, 470-473 (2010).

3. Rogers, C. et al. Closing the Nanographene Gap: Surface-Assisted Synthesis of Peripentacene from 6,6'-Bipentacene Precursors. Angew. Chem. Int. Ed. 54, 15143-15146 (2015).

4. Schleyer, P. V. Introduction: Aromaticity. Chem. Rev. 101, 1115-1117 (2001)

5. Martin, N. \& Scott, L. T. Challenges in aromaticity: 150 years after Kekule's benzene. Chem. Soc. Rev. 44, 6397-6400 (2015).

6. Hückel, E. Quantentheoretische Beiträge zum Benzolproblem. Z. Phys. 70, 204-286 (1931)

7. Frost, A. A. \& Musulin, B. A mnemonic device for molecular orbital energies. J. Chem. Phys. 21, 572-573 (1953).

8. Breslow, R. Antiaromaticity. Acc. Chem. Res. 6, 393-398 (1973).

9. Dias, J. R. \& Aihara, J. Antiaromatic holes in graphene and related graphite defects. Mol. Phys. 107, 71-80 (2009).

10. Jalbout, A. F., Ortiz, Y. P. \& Seligman, T. H. Spontaneous symmetry breaking and strong deformations in metal adsorbed graphene sheets. Chem. Phys. Lett. 564, 69-72 (2013).

11. Wang, H., Maiyalagan, T. \& Wang, X. Review on recent progress in nitrogendoped graphene: synthesis, characterization, and its potential applications. ACS Catalysis 2, 781-794 (2012). 
12. Li, Y. et al. Nitrogen-doped graphene quantum dots with oxygen-rich functional groups. J. Am. Chem. Soc. 134, 15-18 (2012).

13. Vyas, V. S. et al. A tunable azine covalent organic framework platform for visible light-induced hydrogen generation. Nat. Commun. 6, 8508 (2015).

14. Lv, R. et al. Nitrogen-doped graphene: beyond single substitution and enhanced molecular sensing. Sci. Rep. 2, 586 (2012).

15. Liu, Q., Guo, B. D., Rao, Z. Y., Zhang, B. H. \& Gong, J. R. Strong two-photoninduced fluorescence from photostable, biocompatible nitrogen-doped graphene quantum dots for cellular and deep-tissue imaging. Nano Lett. 13 , 2436-2441 (2013).

16. Wang, X. W. et al. Heteroatom-doped graphene materials: syntheses, properties and applications. Chem. Soc. Rev. 43, 7067-7098 (2014).

17. Cai, J. M. et al. Graphene nanoribbon heterojunctions. Nat. Nanotechnol. 9, 896-900 (2014)

18. Vo, T. H. et al. Nitrogen-doping induced self-assembly of graphene nanoribbon-based two-dimensional and three-dimensional metamaterials. Nano Lett. 15, 5770-5777 (2015).

19. Zhang, Y. et al. Direct visualization of atomically precise nitrogen-doped graphene nanoribbons. Appl. Phys. Lett. 105, 023101 (2014).

20. Draper, S. M., Gregg, D. J. \& Madathil, R. Heterosuperbenzenes: A new family of nitrogen-functionalized, graphitic molecules. J. Am. Chem. Soc. 124, 3486-3487 (2002).

21. Takase, M. et al. Pyrrole-fused azacoronene family: the influence of replacement with dialkoxybenzenes on the optical and electronic properties in neutral and oxidized states. J. Am. Chem. Soc. 135, 8031-8040 (2013).

22. Ito, S., Tokimaru, Y. \& Nozaki, K. Benzene-fused azacorannulene bearing an internal nitrogen atom. Angew. Chem. Intl. Ed. 54, 7256-7260 (2015).

23. Yokoi, H. et al. Nitrogen-embedded buckybowl and its assembly with C-60. Nat. Commun. 6, 8215 (2015).

24. Steiner, E., Fowler, P. W., Soncini, A. \& Jenneskens, L. W. Current-density maps as probes of aromaticity: global and Clar $\pi$ ring currents in totally resonant polycyclic aromatic hydrocarbons. Faraday Discuss. 135, 309-323 (2007)

25. Cao, J. et al. The impact of antiaromatic subunits in $[4 n+2] \pi$-systems: bispentalenes with $[4 \mathrm{n}+2] \pi$-electron perimeters and antiaromatic character. $J$. Am. Chem. Soc. 137, 7178-7188 (2015).

26. Berger, R., Wagner, M., Feng, X. L. \& Müllen, K. Polycyclic aromatic azomethine ylides: a unique entry to extended polycyclic heteroaromatics. Chem. Sci. 6, 436-441 (2015).

27. Gross, L., Mohn, F., Moll, N., Liljeroth, P. \& Meyer, G. The chemical structure of a molecule resolved by atomic force microscopy. Science 325, 1110-1114 (2009).

28. Schleyer, P. V., Maerker, C., Dransfeld, A., Jiao, H. J. \& Hommes, N. J. R. V. Nucleus-independent chemical shifts: a simple and efficient aromaticity probe. J. Am. Chem. Soc. 118, 6317-6318 (1996).

29. Berger, R. et al. Synthesis of nitrogen-doped zigzag-edge peripheries: dibenzo9a-azaphenalene as repeating unit. Angew. Chem. Int. Ed. 53, 10520-10524 (2014).

30. Ito, S., Tokimaru, Y. \& Nozaki, K. Isoquinolino[4,3,2-de]phenanthridine: synthesis and its use in 1,3-dipolar cycloadditions to form nitrogen-containing polyaromatic hydrocarbons. Chem. Commun. 51, 221-224 (2015).

31. Wijesinghe, L. P. et al. Methoxy functionalisation: exerting synthetic control of the supramolecular and electronic structure of nitrogen-doped nanographenes. Chem. Commun. 50, 10637-10640 (2014).

32. Wang, X.-Y., Narita, A., Feng, X. \& Müllen, K. $\mathrm{B}_{2} \mathrm{~N}_{2}$-Dibenzo[a,e]pentalenes: effect of the $\mathrm{BN}$ orientation pattern on antiaromaticity and optoelectronic properties. J. Am. Chem. Soc. 137, 7668-7671 (2015).

33. Ishida, M. et al. Protonation-coupled redox reactions in planar antiaromatic meso-pentafluorophenyl-substituted o-phenylene-bridged annulated rosarins. Nat. Chem. 5, 15-20 (2013).

34. Martin-Gago, J. A. On-surface molecular engineering. Nat. Chem. 3, 11-12 (2011).

35. Palma, C.-A. \& Samorì, P. Blueprinting macromolecular electronics. Nat. Chem. 3, 431-436 (2011).

36. Björk, J. \& Hanke, F. Towards design rules for covalent nanostructures on metal surfaces. Chem. Eur. J. 20, 928-934 (2014).

37. Gourdon, A. (ed.). On-Surface Synthesis (Springer International Publishing, Switzerland, 2014).

38. Joshi, S. et al. Boron Nitride on $\mathrm{Cu}(111)$ : An Electronically Corrugated Monolayer. Nano Lett. 12, 5821-5828 (2012).

39. Gross, L. et al. Organic structure determination using atomic-resolution scanning probe microscopy. Nat. Chem. 2, 821-825 (2010).

40. Gross, L. et al. Bond-order discrimination by atomic force microscopy. Science 337, 1326-1329 (2012).

41. Hämäläinen, S. K. et al. Intermolecular contrast in atomic force microscopy images without intermolecular bonds. Phys. Rev. Lett. 113, 186102 (2014).

42. Hapala, P. et al. Mechanism of high-resolution STM/AFM imaging with functionalized tips. Phys. Rev. B 90, 085421 (2014).
43. Moll, N. et al. Image distortions of a partially fluorinated hydrocarbon molecule in atomic force microscopy with carbon monoxide terminated tips. Nano Lett. 14, 6127-6131 (2014).

44. Schuler, B. et al. Contrast formation in Kelvin probe force microscopy of single $\pi$-conjugated molecules. Nano Lett. 14, 3342-3346 (2014).

45. Albrecht, F. et al. Probing charges on the atomic scale by means of atomic microscopy. Phys. Rev. Lett. 115, 076101 (2015).

46. van der Lit, J., Di Cicco, F., Hapala, P., Jelinek, P. \& Swart, I. Submolecular resolution imaging of molecules by atomic force microscopy: the influence of the electrostatic force. Phys. Rev. Lett. 116, 096102 (2016).

47. Nečas, D. \& Klapetek, P. Gwyddion: an open-source software for SPM data analysis. Open Phys. 10, 181-188 (2012).

48. Kresse, G. \& Furthmüller, J. Efficient iterative schemes for ab initio total-energy calculations using a plane-wave basis set. Phys. Rev. B 54, 11169-11186 (1996).

49. Blöchl, P. E. Projector augmented-wave method. Phys. Rev. B 50, 17953-17979 (1994).

50. Dion, M., Rydberg, H., Schröder, E., Langreth, D. C. \& Lundqvist, B. I. Van der Waals density functional for general geometries. Phys. Rev. Lett. 92, 246401 (2004).

51. Hamada, I. van der Waals density functional made accurate. Phys. Rev. B 89, 121103 (2014).

52. Tersoff, J. \& Hamann, D. R. Theory and application for the scanning tunneling microscope. Phys. Rev. Lett. 50, 1998-2001 (1983).

53. Lorente, N. \& Persson, M. Theoretical aspects of tunneling-current-induced bond excitation and breaking at surfaces. Faraday Discuss. 117, 277-290 (2000).

\section{Acknowledgements}

C.-A.P. was supported from the European Union's Horizon 2020 research and innovation program 2D ink (no. 664878). X.-Y.W. is grateful to a fellowship from the Alexander von Humboldt Foundation. R.B. appreciates support by European Social Fund and the Federal State of Saxony (ESF-Project "GRAPHD"). M.G. acknowledges the H2020-MSCA-IF-2014 program and W.A. a Heisenberg professorship by the DFG. Dr Tilo Lübken is acknowledged for help in NMR measurements. This work was partially supported by the ERC Consolidator Grant NanoSurfs (no. 615233), the Max Planck Society, the German Excellence Initiative "Center for Advancing Electronics Dresden" (cfaed), and the Graphene Flagship.

\section{Author contributions}

X.-Y.W., M.R., Y.H., J.B. and A.R. contributed equally to this work. X.-Y.W., M.R., R.R and R.B. performed the solution experiments and related calculations. Y.H., A.R., M.G. and C.-A.P. performed the experiments at surfaces. J.B. performed the DFT simulations at interfaces and C.-A.P. the AFM simulations. F.H. and J.J.W. characterized the crystals W.A. and J.V.B. designed and conceived the UHV methods. A.N., R.B., X.F., C.-A.P. and K.M. conceived and designed the experiments. All the authors contributed to the manuscript.

\section{Additional information}

Supplementary Information accompanies this paper at doi: 10.1038/s41467-017-01934-1.

Competing interests: The authors declare no competing financial interests.

Reprints and permission information is available online at http://npg.nature.com/ reprintsandpermissions/

Publisher's note: Springer Nature remains neutral with regard to jurisdictional claims in published maps and institutional affiliations.

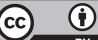

Open Access This article is licensed under a Creative Commons Attribution 4.0 International License, which permits use, sharing, adaptation, distribution and reproduction in any medium or format, as long as you give appropriate credit to the original author(s) and the source, provide a link to the Creative Commons license, and indicate if changes were made. The images or other third party material in this article are included in the article's Creative Commonslicense, unless indicated otherwise in a credit line to the material. If material is not included in the article'sCreative Commons license and your intended use is not permitted by statutory regulation or exceeds the permitted use, you will need to obtain permission directly from the copyright holder. To view a copy of this license, visit http://creativecommons.org/ licenses/by/4.0/

(C) The Author(s) 2017 